\title{
Gestión de mantenimiento y producción más limpia en tres instituciones de salud de Medellín, Colombia
}

\author{
W. Orozco ${ }^{1, \psi}$, G. Narváez ${ }^{2}$, W. García Gómez ${ }^{3}$, A. Quintero Rodas ${ }^{4}$ \\ ${ }^{1}$ Grupo de Investigación e Innovación en Energías GIIEN. Facultad de Ingeniería, \\ Institución Universitaria Pascual Bravo. Medellín, Colombia \\ ${ }^{2}$ Grupo de Investigación ALQUIMIA. Facultad de Ciencias Exactas y Aplicadas, \\ Instituto Tecnológico Metropolitano. Medellín, Colombia \\ ${ }^{3,4}$ Grupo de Investigación e Innovación Biomédica GI2B. Facultad de Ciencias Exactas y Aplicadas, \\ Instituto Tecnológico Metropolitano. Medellín, Colombia
}

\begin{abstract}
Resumen - Objetivo: Realizar un diagnóstico del estado de la gestión del mantenimiento de equipos biomédicos en el área de urgencias de tres (3) instituciones de nivel tres de complejidad médica de la ciudad de Medellín, Colombia, dentro del proyecto de investigación producción más limpia en salud. Metodología: Se diseñó una encuesta a partir de la Guía Sectorial de Producción más Limpia, en hospitales, clínicas y centros de salud, donde se obtuvo una lista de chequeo para la revisión del estado de la gestión de mantenimiento de los equipos biomédicos más complejos utilizados en urgencias. Resultados: La gestión de mantenimiento de los equipos biomédicos más complejos es muy buena en el hospital 1 y excelente en los hospitales 2 y 3; la metrología y gestión de mantenimiento en los equipos biomédicos del área de urgencias de los tres hospitales es excelente. Conclusión: La gestión de mantenimiento es muy importante para el ahorro económico en las instituciones, ya que se pueden reducir tiempos muertos de los equipos sin afectar la prestación de los servicios, aparte de que se reducen gastos en la compra de repuestos, y generación de residuos causantes de impactos negativos al medio ambiente.
\end{abstract}

Palabras clave - Equipos biomédicos, gestión de mantenimiento, hospitales, metrología biomédica, producción más limpia.

\section{Cleaner Maintenance and Production Management in Three Health Institutions in Medellín, Colombia}

\footnotetext{
Abstract - Objective: to perform a diagnosis of the state of biomedical equipment maintenance management in the emergency areas of three (3) institutions of level three of medical complexity in the city of Medellín, Colombia. The realm is within the cleaner production in health research project. Methodology. A survey was designed based on the Cleaner Production Sector. Guide: in
}

\% Dirección para correspondencia: william.orozco@pascualbravo.edu.co

DOI: https://doi.org/10.24050/19099762.n21.2017.1168 
hospitals, clinics and health centers, where a check list was obtained to review the state of maintenance management of the most complex biomedical equipment used in the emergency room. Results: maintenance management of the most complex biomedical equipment is very good in hospital 1 and excellent in hospitals 2 and 3. Metrology and maintenance management in the biomedical equipment of the emergency area of all three hospitals is excellent. Conclusion: maintenance management is very important for financial savings in institutions, since they can thus reduce equipment downtime without hindering the provision of services, along with reducing costs in the purchase of spare parts and generation of waste, which causes a negative impact on the environment.

Keywords - Biomedical equipment, maintenance management, hospitals, biomedical metrology, cleaner production.

\section{Gestão de MANUTENÇÃO E PRODUÇÃO MAIS LIMPA EM TRÊS INSTITUIÇÕES DE SAÚde de Medellín, ColÔMBIA}

Resumo-Objetivo: Realizar um diagnóstico do estado da gestão da manutenção de equipamentos biomédicos na área de urgências de três (3) instituições de nível três de complexidade médica da cidade de Medellín, Colômbia, dentro do projeto de investigação produção mais limpa em saúde. Metodologia: Desenhou-se uma pesquisa a partir da Guia Setorial de Produção mais limpa, em hospitais, clínicas e centros de saúde, onde se obteve uma lista de verificação para a revisão do estado da gestão de manutenção dos equipamentos biomédicos mais complexos utilizados em urgências. Resultados: A gestão de manutenção dos equipamentos biomédicos mais complexos é muito boa no hospital 1 e excelente nos hospitais 2 e 3; a metrologia e gestão de manutenção nos equipamentos biomédicos da área de urgências dos três hospitais é excelente. Conclusão: A gestão de manutenção é importantíssima para a poupança económica nas instituições, já que podem-se reduzir tempos mortos dos equipamentos sem afetar a prestação dos serviços, aparte de que se reduzem despesas na compra de repostos, e geração de resíduos causantes de impactos negativos ao meio ambiente.

Palavras-chave-Equipamentos biomédicos, gestão de manutenção, hospitais, metrologia biomédica, produção mais limpa.

\section{INTRODUCCIÓN}

L as instituciones prestadoras del servicio de salud, además de las intervenciones para transformar las enfermedades en bienestar a las personas, son generadoras de una serie de impactos negativos ocasionados desde los diferentes procesos e insumos que se utilizan para llevar a cabo los procedimientos de atención médica y las demás actividades que soportan el objeto y la naturaleza social [1].

La implementación de mejores prácticas de responsabilidad social y ambiental en Clínicas y Hospitales del Clúster de Salud de Medellín, orientadas desde la Producción Limpia y el Consumo Sostenible (PL\&CS), es ratificado en Colombia desde el año 2010 a través de la Política Nacional de Producción y Consumo Sostenible [2], con el objeto de mejorar las prácticas de responsabilidad corporativa en empresas del sector público y privado, generar valor agregado desde la producción y el consumo sostenible de bienes y servicios, hacer un uso racional de los recursos naturales, prevenir riesgos y peligros que afecten la salud humana y el medio ambiente, e incrementar la productividad y la competitividad en las empresas de los distintos sectores económicos del país a nivel nacional $\mathrm{y}$ en los mercados internacionales [3].

La gestión del mantenimiento hoy en día se está trabajando con procesos de producción más limpia [4], que tie- nen como objetivo la reducción de impactos en el ambiente, minimización de riesgos para los trabajadores, además, disminuir costos incurridos en la producción de residuos y emisiones a la atmósfera [5]. En consecuencia, con lo planteado anteriormente desde el año 2013 se viene realizando por parte del Instituto Tecnológico Metropolitano (ITM), de la Institución Universitaria Pascual Bravo (IU Pascual Bravo), un estudio acerca del estado de la gestión de mantenimiento en el servicio de urgencias de tres instituciones de salud de niveles III y IV del área metropolitana de la ciudad de Medellín, Colombia, en el contexto del proyecto de investigación de producción más limpia en salud.

\section{MetodologíA}

Se diseñó una encuesta teniendo en cuenta la Guía Sectorial de Producción más Limpia, en instituciones de salud y de la resolución de habilitación 1441 de 2014 [1], [6], donde se elaboró una lista de chequeo para la revisión del estado de la gestión de mantenimiento de los equipos biomédicos de alto y mediano riesgo utilizados en el servicio de urgencias (monitores de signos vitales, desfibriladores, tensiómetros, servocunas, electrocardiógrafos y ventiladores mecánicos); Tabla I. Para esto la lista de chequeo fue dividida en dos grandes áreas principales; mantenimiento de los equipos biomédicos utilizados con 
14 registros enfocadas a definir la existencia de protocolos de mantenimiento, tipos de mantenimientos empleados, personal encargado del mantenimiento, hojas de vida de los equipos, órdenes de trabajo, planes y programas de mantenimiento y hojas de registro de los equipos.

Y metrología y gestión de mantenimiento de los equipos biomédicos con 12 registros para determinar la forma como se articula y coordina la calibración de los equipos con el mantenimiento de éstos, la entidad o entidades que realizan la calibración de los equipos, el personal encargado de la gestión de mantenimiento, el plan presupuestal para realizar el mantenimiento y los planes de habilitación, certificación y acreditación que tiene la institución de salud definidos para los próximos años [6].

Luego a cada registro de la lista de chequeo se le asigna una valoración de 0 a 10 de acuerdo a los siguientes criterios: Tabla 1.

C: Cumple totalmente (10 puntos)

N.C: No Cumple (0 puntos)

C.P: Cumple Parcialmente (5 puntos)

Tabla 1. Listado de verificación para la gestión de mantenimiento

\begin{tabular}{c|c|c|c|c|c}
\hline \multirow{2}{*}{ (pts.) } & \multicolumn{4}{|c}{ Listado de Verificación } \\
\cline { 2 - 6 } 1 & Parámetros & C & N.C & C.P & Observaciones \\
\hline \multirow{2}{*}{2} & $\begin{array}{c}\text { Mantenimiento de los } \\
\text { equipos biomédicos } \\
\text { utilizados en } \\
\text { urgencias }\end{array}$ & & & & \\
\hline \multirow{2}{*}{2} & $\begin{array}{c}\text { Metrología y gestión } \\
\text { de mantenimiento } \\
\text { en los equipos } \\
\text { biomédicos del área } \\
\text { de urgencias }\end{array}$ & & & & \\
\hline
\end{tabular}

Para obtener una valoración cualitativa y porcentual por cada área evaluada en las tres instituciones de salud seleccionadas para el diagnóstico, como lo muestra la Tabla 2.

Finalmente se establecen las fortalezas (F), oportunidades de mejora (O.M) y se realizan recomendaciones (R) a las instituciones de salud.

Tabla 2. Valoración cualitativa y porcentual por cada área evaluada

\begin{tabular}{c|c|c}
\hline \multirow{2}{*}{ (pts.) } & \multicolumn{2}{|c}{ Valoración } \\
\cline { 2 - 3 } & Cualitativa & Porcentual (\%) \\
\hline 1 & No se tiene, o no se cumple & 0 \\
\hline 2 & Insuficiente & 0 a 30 \\
\hline 3 & Aceptable & 31 a 50 \\
\hline 4 & Bueno & 51 a 70 \\
\hline 5 & Muy bueno & 71 a 90 \\
\hline 6 & Excelente & 91 a 100 \\
\hline
\end{tabular}

\section{RESULTADOS}

De acuerdo con los parámetros establecidos en la Tabla 2, se obtuvieron los resultados presentados en la Tabla 3, lo cual permite identificar que las entidades evaluadas, cuentan con sistemas de planificación del mantenimiento y la calibración de los equipos biomédicos. Cabe resaltar que, la calibración de los equipos biomédicos en algunas ocasiones es realizada por la institución, mientras que en otras este servicio es prestado por empresas externas, lo cual está sujeto a la capacidad institucional y las características de los equipos. Además, dentro de la revisión se pudo encontrar que los departamentos de ingeniería biomédica se encargan también del mantenimiento de la infraestructura hospitalaria.

Tabla 3. Comparación de resultados de las tres instituciones evaluadas

\begin{tabular}{c|c|c|c|c}
\hline \multicolumn{5}{c}{ Valoración Hospital } \\
\hline & & 1 & 2 & 3 \\
\hline \multirow{2}{*}{ Cualitativa } & G-Mtto & Muy bien & Excelente & Excelente \\
\cline { 3 - 5 } & Mtg & Excelente & Excelente & Excelente \\
\hline \multirow{3}{*}{$\begin{array}{c}\text { Porcentual } \\
(\%)\end{array}$} & G-Mtto & 86 & 92 & 92 \\
\cline { 2 - 5 } & Mtg & 96 & 100 & 100 \\
\hline
\end{tabular}

G-Mtto: mantenimiento de los equipos biomédicos, Mtg: metrología y gestión de mantenimiento de los equipos biomédicos.

La Gestión de Mantenimiento de los equipos biomédicos utilizados en urgencias en la institución 1 es muy buena con una valoración del $86 \%$. Este resultado, a pesar de ser competitivo en el medio, hace un llamado de atención para el hospital, para que a través de la autoevaluación y de mejoras internas, logren subir dichos indicadores y prestar un servicio de calidad y responsabilidad. Dicho valor es obtenido del análisis de la Tabla 2 y de la lista de chequeo aplicada a algunos grupos de equipos de alto riesgo (monitores de signos vitales, desfibriladores, tensiómetros, servocunas, electrocardiógrafos y ventiladores mecánicos) del servicio de urgencias; Fig. 1.

La Metrología y Gestión de Mantenimiento en los equipos biomédicos del área de urgencias es excelente con una valoración del 96\%. Valor obtenido de la Tabla 2 y del análisis del instructivo aplicado a los grupos de equipos mencionados anteriormente, Fig. 1.

La Gestión de Mantenimiento de los equipos biomédicos utilizados en urgencias en los Hospitales 2 y 3 es excelente con una valoración del $92 \%$. Es de resaltar que el resultado de $92 \%$ para la gestión del mantenimiento, es porque se desconoce el modelo de mantenimiento productivo total (TPM), y el mantenimiento centrado en confiabilidad (RCM); esto no implica que existan fallas 
graves en el proceso de gestión del mantenimiento, sino que dejan de utilizar estrategias modernas del mantenimiento que ayudarían a que el proceso fuera más rápido y eficiente. La aplicación de las estrategias mencionadas anteriormente, podrían mejorar no solo los resultados del estudio sino también, la productividad y el servicio que se presta en el hospital.

La Metrología y Gestión de Mantenimiento en los equipos biomédicos en el servicio de urgencias es excelente con una valoración del 100\%. Valor obtenido para algunos equipos de alto y mediano riesgo (monitores de signos vitales, desfibriladores, tensiómetros, servocunas, electrocardiógrafos y ventiladores mecánicos) del servicio de urgencias, Tabla 2 y Fig.1.

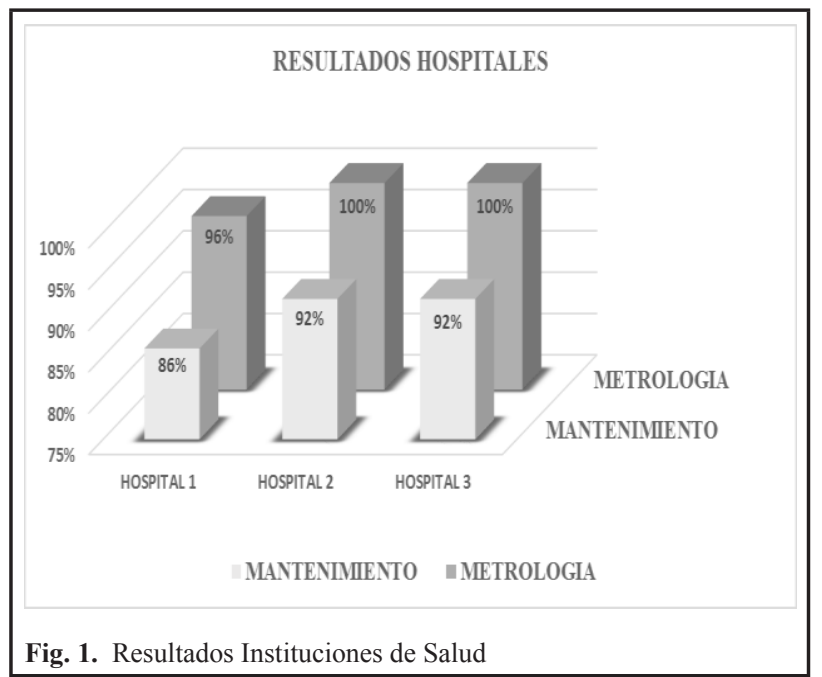

La Fig. 1 y la Tabla 2 muestra que en estas tres instituciones de salud hay una muy buena gestión de mantenimiento de los equipos, lo cual reduce considerablemente el número de paros de emergencia de los equipos de alto, mediano y bajo riesgo utilizados en el servicio de urgencias así como los repuestos utilizados, por lo tanto, se disminuyen los contaminantes y residuos generados por esta actividad, ayudando así a la producción más limpia en el área de la salud y a la conservación del planeta [7].

En general, las instituciones presentan, según el listado de verificación diseñado para la recolección de la información, una gestión de mantenimiento, que, si bien puede mejorar, es competitiva en el medio, demostrando la capacidad y disposición de adoptar nuevas mejoras dentro de la planificación de la gestión de mantenimiento y la metrología, siendo de gran importancia el personal que contratan, la tecnología que se adquiere y el costo efectivo de la misma, para así prolongarle la confiabilidad técnica.

\section{DiscusióN}

La gestión de mantenimiento es una metodología científicamente probada que integra los esfuerzos de varias áreas de la institución utilizando racionalmente los recursos, planificando, controlando, y guiando la organización hacia sus fines y objetivos; además permite optimizar el uso de las herramientas tecnológicas existentes, así como el tiempo de ejecución de las diferentes actividades realizadas por los operarios, técnicos y personal asistencial, con el fin de garantizar que los diferentes equipos y dispositivos de ayuda médica reciban los mantenimientos correctivos y preventivos necesarios en el momento oportuno [8].

Lo anterior permite un mayor rendimiento y confiabilidad de los equipos y dispositivos médicos, lo que incrementará su nivel de calidad, disponibilidad y seguridad mejorando así la prestación del servicio de las instituciones de salud, al tener siempre información actualizada, oportuna y veraz de cada uno de los equipos, lo que permite tomar decisiones acertadas a la hora de los mantenimientos correctivos o preventivos y/o reposición de accesorios de la tecnología médica [9].

Como se muestra en los resultados, de la valoración porcentual de los hallazgos por cada área evaluada en mantenimiento de los equipos biomédicos utilizados en el servicio de urgencias y la metrología y gestión de mantenimiento de los equipos biomédicos; es necesario implementar planes de mejoramiento en algunos aspectos, tales como: la capacitación del personal en el uso de herramientas y conceptos de las tendencias modernas del mantenimiento, con el fin de canalizarlos adecuadamente, para prestar un servicio de salud de calidad a costos razonables [10].

La salud pública y privada requieren estándares de calidad para el seguimiento, control y transparencia en la prestación de servicios de salud [11], de forma que permita ganar paulatinamente la confianza de los diferentes usuarios y mejorar la imagen que se tiene de la gestión del mantenimiento de equipo biomédico en el área de urgencias en las entidades de salud, lo cual generaría una percepción de seguridad y confianza en la población de interés que usa los diferentes equipos y servicios [12].

\section{Conclusiones}

La gestión de mantenimiento es muy importante en las instituciones de salud para conservar los inmuebles, equipos, instalaciones, herramientas, en condiciones de funcionamiento seguro, eficiente y económico, previniendo daños o reparándolos cuando ya se hubieran producido, con lo anterior se logra el ahorro económico de repuestos, insumos, mano de obra directa e indirecta, mantenimientos 
de urgencias, etc, ya que se pueden reducir tiempos muertos de los equipos sin afectar la prestación de los servicios, aparte que se aumenta la confiabilidad y disponibilidad de los equipos, y finalmente se reduce también la generación de residuos y contaminantes causantes de impactos negativos al medio ambiente.

Teniendo en cuenta la resolución 1441 de 2014 del Ministerio de Salud y Protección Social, por la cual se define los procedimientos y condiciones que debe cumplir los Prestadores de Servicio de Salud para habilitar los servicios, se encontró que el área de urgencias de las instituciones encuestadas cuentan con los grupos de equipos de alto y mediano riesgo necesarios para prestar el servicio adecuadamente, la gestión de mantenimiento de los equipos biomédicos más complejos es muy buena en el hospital 1 y excelente en los hospitales 2 y 3 ; la metrología y gestión de mantenimiento en los equipos biomédicos del área de urgencias de los tres hospitales es excelente, Tabla 2 y Fig 1.

\section{REFERENCIAS}

[1]. (2011) Guía Sectorial de Producción más Limpia, hospitales, clínicas y centros de salud, Disponible http://www.cnpml. org/templates/panalVerde/images/publicaciones/GuiaSect orialDePMLEnHospitalesClinicasyCentrosDeSalud.pdf

[2]. J. Narváez, L. Sepúlveda, A. Ramírez, W. Orozco, J. Moreno. "Producción más limpia en el sector salud", Memorias Convención Internacional de Salud Pública. La Habana 3-7 de diciembre de 2012.

[3]. (2011). C.M. Herrera. La evolución y el futuro de la producción más limpia en Colombia. [Consultado]. 25(9). Disponible en: http://revistaing.uniandes.edu.co/pdf/26a12.pdf

[4]. W. Orozco, G. Narváez. Maintenance Management in Biomedical Equipments in the Context of the Research Project Cleaner Production in the Health Cluster of Medellín, Colombia. Revista Ingeniería Biomédica, vol. 9 pp. 15-19, 2015

[5]. R. Luken, R. Van, H. Leuenberger, and P. Schwager. A 20year retrospective of the National Cleaner Production Centres programme, Journal of Cleaner Production, vol. 112, pp.1165$1174,2016$.

[6]. Minprotecciónsocial. Resolución 1441, procedimientos y condiciones que debe cumplir los Prestadores de Servicio de Salud para habilitar los servicios. Santa Fe de Bogotá: Supersalud, 2014.

[7]. W. Martinez. Gerencia del mantenimiento en hospitales. Maintenance Management Healthcare Administration. Honolulu, Hawai: Atlantic International University septiembre 2009; p 7.

[8]. C. Vásquez and M. Pérez. Guía para la implementación del Programa Nacional de Tecnovigilancia en el Hospital General de Medellín. Rev. Ingeniería Biomédica, Medellín, Colombia, vol 5, no 9, pp 60-78. jan-jun, 2011.

[9]. W. Orozco, F. Cortés Caracterización de la gestión del mantenimiento de equipo biomédico en servicios de urgencia de clínicas y hospitales de Medellín en el período 2008-2009. En: Rev Cienc Salud, vol 11, no 1, pp3544, 2013.
[10]. A.M Cruz, W.A Aguilera and D.A Días. A comparative study of maintenance services using the data-mining technique. Rev Salud Pública, Bogotá, vol 11, no 4, pp 653-61. Aug 2010b

[11]. M. Carnero. Simulation of trends of maintenance policies. A case study in a Hospital. 7th WSEAS Int. Conf. on applied computer \& applied computational science (ACACOS '08), Hangzhou, China 2008; April 6-8.

[12]. A. Muñoz. Gestión de mantenimiento en la Clínica el Prado Santa Marta, Colombia. Santiago de Cali: Universidad Autónoma de Occidente; 2008; p. 105. 